\title{
A Retrospective Analysis of Clinical Utility of AJCC 8th Edition Cancer Staging System for Breast Cancer
}

\author{
Hui Hu${ }^{\mathrm{a}}$, Wei Wei ${ }^{\mathrm{a}}$, Xin $\mathrm{Yi}^{\mathrm{a}}$, Ling Xin ${ }^{\mathrm{b}}$, Yinhua Liu ${ }^{\mathrm{b}, \mathrm{c}}$
}

\begin{abstract}
Background: The aim was to study the clinical utility of AJCC 8th edition prognostic stage system for patients with breast cancer.

Methods: According to the AJCC 8th edition cancer staging system, a total of 784 patients with breast cancer diagnosed by Department of Breast Surgery in Peking University Shenzhen Hospital from January 2011 to June 2016 were analyzed in anatomic and prognostic stage.

Results: Five hundred and five (64.4\%) patients prognosis staging changed compared with anatomic stage. Among them, $63(33.5 \%)$ patients were in stage I, $295(73.8 \%)$ patients in stage II, and 128 $(72.7 \%)$ patients in stage III according to anatomic stage, respectively. The distribution and variety of the anatomic and prognostic stage differ in intrinsic subtypes of breast cancer $(\mathrm{P}<0.001)$.
\end{abstract}

Conclusion: AJCC 8th edition cancer staging system is an important prognostic factor for disease outcome of breast cancer.

Keywords: Breast cancer; AJCC breast cancer staging system; Prognostic staging

\section{Introduction}

AJCC 8th edition cancer staging system will be implemented since January 1, 2018 [1]. For the first time, AJCC demonstrated prognostic stage group to anatomic cancer stage system, aimed to provide a reasonable reference in clinical decisionmaking. According to the prognostic stage system, patients diagnosed with breast cancer in Department of Breast Surgery, Peking University Shenzhen Hospital from 2011 to 2016 were

Manuscript accepted for publication April 26, 2017

${ }^{a}$ Department of Breast Surgery, Peking University Shenzhen Hospital, Shenzhen, Guangdong, China

${ }^{b}$ Breast Disease Center, Peking University First Hospital, XiShiKu Street, Beijing, China

${ }^{\mathrm{c} C}$ Corresponding Author: Liu Yinhua, Breast Disease Center, Peking University First Hospital, No.8 XiShiKu Street, Beijing 100034, China.

Email: liuyinhua@medmail.com.cn

doi: https://doi.org/10.14740/wjon1039e re-evaluated and the clinical value of prognostic stage system and clinicopathological classification was analyzed [2].

\section{Methods}

A retrospective analysis was conducted to patients with invasive breast cancer diagnosed by Department of Breast Surgery, Peking University Shenzhen Hospital from January 2011 to June 2016. Clinical data and pathological information were available. All pathologic specimens were reviewed by two experienced pathologists who provided following pathological information: primary tumor size, tumor grade, and immunohistochemistry (IHC) results including expression of estrogen receptor (ER), progesterone receptor (PR), human epidermal growth factor-2 (HER2) and Ki67 expression, demographic features and clinicopathological classification.

According to the definition of St.Gallen Breast Cancer consensus in 2011: luminal A (ER and/or PR positive, HER2 negative and low Ki67), luminal B HER2 negative (ER and/or PR positive, HER2 negative and high Ki67 $\geq 15 \%$ ), luminal B HER2 enriched (ER and/or PR positive, HER2 positive), HER2 positive (ER and PR negative and HER2 positive) and triple negative (ER, PR and HER2 negative) [3].

H\&E staining immunohistochemical examination was routinely performed on tumor specimens. Pathological results were re-checked according to the American Society of Clinical Oncology/College of American Pathologists (ASCO/CAP) Guideline for Breast Cancer immunohistochemical testing of ER and PR in 2010 and Guideline for human epidermal growth factor receptor 2 testing in 2013 [4]. Histologic grade of invasive breast cancer was assessed according to Nottingham Grading System (NGS), which is based on tubule formation, nuclear grade and the mitotic rate [5]. Status of hormone receptor (HR) was considered positive if at least $1 \%$ positive tumor nuclei were detected in the samples. HER2 was determined in all pathological specimens with invasive breast cancer. Testing criteria define HER2-positive status when (on observing within an area of tumor that amounts to $>10 \%$ of contiguous and homogeneous tumor cells) there is evidence of protein overexpression (IHC) or gene amplification (HER2 copy number or HER2/CEP17 ratio by FISH based on counting at least 20 cells within the area).

Anatomic and prognostic staging of patients with breast cancer was evaluated by AJCC 8th cancer staging system [1].

T-test was used to determine the statistic difference be- 
tween anatomic and prognostic cancer stage in different subtypes. All statistical tests and P-values were two-tailed, and P $<0.05$ was considered statistically significant. Analysis was conducted with SPSS software version 20.0 (IBM-SPSS, Chicago, IL, USA).

\section{Results}

A total of 784 patients newly diagnosed with invasive breast cancer in the Department of Breast Surgery, Peking University Shenzhen Hospital from January 2011 to June 2016 were enrolled in this study. All of patients were female, the median age of breast cancer diagnosis was 46.4 years old (18 - 84 years old); 686 (87.5\%) were premenopausal, and 98 (12.5\%) were postmenopausal (Table 1). There was no statistic significance between 8th and 7th AJCC anatomic cancer staging system for breast cancer $[1,6]$. Prognostic stage information included: histological grade $(\mathrm{G})$, human epidermal growth factor receptor-2 (HER2), estrogen receptor (ER) and progesterone receptor (PR) $[7,8]$.

As for anatomic stage, $188(24.0 \%)$ patients were stage I, totally IA. Four hundred (51\%) patients were stage II, of which $243(31.0 \%)$ were IIA and $157(20.0 \%)$ were IIB; $176(22.4 \%)$ cases of stage III included $102(13.0 \%)$ patients in IIIA, seven $(0.9 \%)$ in IIIB, and $67(8.5 \%)$ in IIIC. Twenty $(2.6 \%)$ cases were stage IV (Table 2).

As for prognostic stage, $337(42.9 \%)$ cases were stage I, of which $125(15.9 \%)$ cases were in IA, and $212(27.0 \%)$ were in IB. One hundred and forty-five $(18.5 \%)$ cases were in stage II, with $54(6.8 \%)$ in IIA and $91(11.6 \%)$ in IIB. Two hundred and five $(26.1 \%)$ cases were in stage III, of which $82(10.5 \%)$ were in IIIA, $67(8.5 \%)$ in IIIB and $56(7.1 \%)$ cases in IIIC. Twenty $(2.6 \%)$ cases were in stage IV as the prognostic stage system showed (Table 2).

In addition to TNM stage IV, prognostic stage also changed in anatomic stage I-III. Among them, 63 (33.5\%) patients in anatomic stage IA upstaged to prognostic stage IIA $(21 / 63$, $11.2 \%)$ and IIB (42/63, 22.3\%). Seventy-six (31.3\%) patients in stage IIA upstaged to IIB $(32 / 76,13.2 \%)$ and IIIA $(44 / 76$, $18.1 \%)$. One hundred and thirty-nine $(57.2 \%)$ patients in stage IIA downstaged to IB. Eighteen $(11.5 \%)$ patients in stage IIB downstaged to IB $(17 / 18,10.8 \%)$ and IIA $(1 / 18,0.6 \%)$. Sixtytwo $(39.5 \%)$ patients in stage IIB downstaged to stage IIIA $(25 / 62,5.9 \%)$, IIIB $(23 / 62,14.6 \%)$ and IIIC (14/62, 8.9\%). Seventy-seven patients (all anatomic stage IIB) could not be restaged in prognostic stage (Table 3 ).

Of all patients enrolled, patients with luminal A, luminal B, HER2-enriched and triple negative were 88 (11.2\%), 515 (65.7\%), 77 (9.8\%) and 104 (13.3\%). The distribution of patients in prognostic stage was significantly different among the five subtypes of breast cancer $(\mathrm{P}<0.001)$.

\section{Discussion}

On October 6, 2016, the AJCC 8th edition of cancer staging system was updated, and it will be implemented globally in
Table 1. Patients and Tumor Characteristics

\begin{tabular}{|c|c|c|}
\hline Characteristic & $\mathbf{N}$ & $\%$ \\
\hline \multicolumn{3}{|l|}{ Age, years } \\
\hline$<35$ & 121 & $15.5 \%$ \\
\hline$\geq 35$ & 663 & $84.5 \%$ \\
\hline \multicolumn{3}{|l|}{ Menopausal status } \\
\hline Postmenopausal & 98 & $12.5 \%$ \\
\hline Premenopausal & 686 & $87.5 \%$ \\
\hline \multicolumn{3}{|l|}{$\mathrm{T}$ stage at diagnosis } \\
\hline $\mathrm{T} 1$ & 292 & $37.2 \%$ \\
\hline $\mathrm{T} 2$ & 439 & $56.0 \%$ \\
\hline $\mathrm{T} 3$ & 35 & $4.5 \%$ \\
\hline $\mathrm{T} 4$ & 18 & $2.3 \%$ \\
\hline \multicolumn{3}{|l|}{$\mathrm{N}$ status at diagnosis } \\
\hline N0 & 381 & $48.6 \%$ \\
\hline N1 & 223 & $28.4 \%$ \\
\hline N2 & 105 & $13.4 \%$ \\
\hline N3 & 75 & $9.6 \%$ \\
\hline \multicolumn{3}{|l|}{ M status at diagnosis } \\
\hline M0 & 764 & $97.4 \%$ \\
\hline M1 & 20 & $2.6 \%$ \\
\hline \multicolumn{3}{|l|}{ ER status } \\
\hline Positive & 594 & $75.8 \%$ \\
\hline Negative & 190 & 24.2 \\
\hline \multicolumn{3}{|l|}{ PR status } \\
\hline Positive & 513 & $65.4 \%$ \\
\hline Negative & 217 & $34.6 \%$ \\
\hline \multicolumn{3}{|l|}{ HER2 status } \\
\hline Positive & 238 & $30.4 \%$ \\
\hline Negative & 546 & $69.6 \%$ \\
\hline
\end{tabular}

2018. Although the new cancer stage system put forward prognostic stage groups as a crucial part of cancer stage system, it is still established on the foundation of primary tumor (T), lymph node $(\mathrm{N})$ and metastasis $(\mathrm{M})[1,9]$.

Our study respectively analyzed the clinicopathological characteristics of anatomic and prognostic stage of 784 patients who were newly diagnosed with invasive breast cancer in Department of Breast Surgery, Peking University Shenzhen Hospital from January 2011 to June 2016. Patients in anatomic stage IV remain stage IV in prognostic stage, indicating patients with distant metastasis had poor prognosis regardless of other biological characteristics of tumor. Patients in the same anatomic stage, but with different immunochemistry results may have different prognostic stage. AJCC 8th edition cancer stage system definitely provides a new way to value disease outcomes and help to make treatment decisions, which is more objective and rigorous than any other methods in clinic ap- 
Table 2. Distribution of the Prognostic Stage and TNM Stage of the Patients

\begin{tabular}{lllllllllll}
\hline \multirow{2}{*}{$\begin{array}{l}\text { TNM } \\
\text { stage }\end{array}$} & IA & IB & IIA & IIB & IIIA & IIIB & IIIC & IV & Unknown & Total \\
\hline IA & 125 & 42 & 21 & 0 & 0 & 0 & 0 & 0 & 0 & $188(24.0 \%)$ \\
IIA & 0 & 139 & 28 & 32 & 44 & 0 & 0 & 0 & 0 & $243(31.0 \%)$ \\
IIB & 0 & 17 & 1 & 0 & 25 & 23 & 14 & 0 & 77 & $157(20.0 \%)$ \\
IIIA & 0 & 14 & 4 & 59 & 11 & 10 & 4 & 0 & 0 & $102(13.0 \%)$ \\
IIIB & 0 & 0 & 0 & 0 & 0 & 3 & 4 & 0 & 0 & $7(0.9 \%)$ \\
IIIC & 0 & 0 & 0 & 0 & 2 & 31 & 34 & 0 & 0 & $67(8.5 \%)$ \\
IV & 0 & 0 & 0 & 0 & 0 & 0 & 0 & 20 & 0 & $20(2.6 \%)$ \\
Total (\%) & $125(15.9 \%)$ & $212(27.0 \%)$ & $54(6.9 \%)$ & $91(11.6 \%)$ & $82(10.5 \%)$ & $67(8.5 \%)$ & $56(7.1 \%)$ & $20(2.6 \%)$ & $77(9.8 \%)$ \\
\hline
\end{tabular}

plication.

Patients in anatomic stage I, II, III account for $24 \%, 51 \%$ and $22.5 \%$ of all patients enrolled, while patients in prognostic stage I, II, III account for $42.9 \%, 18.5 \%$ and $26.1 \%$. Compared to prognostic stage system, the cancer stage of patients in stage I upstaged significantly, and the proportion of stage II downstaged significantly. On the contrast, patients in anatomic stage III almost remained the same stage in prognostic stage system. The result indicated that most patients in anatomic stage II had better disease outcome than we supposed when took biological information into consideration. Among them, $57.2 \%$ patients in anatomic stage IIA downstaged to stage IB and $11.5 \%$ of those in stage IIB downstaged to stage IB. Therefore, patients downstaged in prognostic stage need to be personalized when making physical decision to avoid overtreatment. For patients in anatomic stage II and III, the combined treatment of patients with advanced stage in prognostic stage should be emphasized.

The clinicopathological classification was proposed in the St.Gallen consensus report 2011, to classify the subtype of breast cancer and has been widely recognized. According to the consensus, patients with luminal A, luminal B, HER2enriched and triple negative were $88(11.2 \%), 515(65.7 \%)$, $77(9.8 \%)$ and $104(13.3 \%)$. The result is approximate to other studies $[10,11]$. Intrinsic subtypes of breast cancer were different in the distribution and variety of anatomic staging and prognostic stage, and the difference was statistically significant $(\mathrm{P}<0.001)$ (Table 4$)$.

The study showed that the majority of luminal type breast cancer downstaged in prognostic stage. Among them, the prognostic stage of luminal A breast cancer was downstaged in $44.3 \%$ of patients, and only $1.1 \%$ of the patients upstaged; luminal B HER2 positive downstaged in $54.1 \%$ patients, and $22 \%$ of the patients upstaged; luminal B HER 2 negative downstaged in $39.9 \%$ cases, and $13.5 \%$ of the patients upstaged. No patient downstaged in both HER2 positive subtype and triple negative subtype, and the proportions of patients with upstaged prognosis stage in these two subtypes were $91.3 \%$ and $68.8 \%$, respectively. The highest proportion of prognostic stage I and II was luminal A which was $87.4 \%$. While the prognostic stage I, II proportion accounted for $69.2 \%$ and $67.1 \%$, respectively, and prognostic stage III, IV proportion accounted for $22.7 \%$ and $17.9 \%$, respectively in luminal B HER2 positive and negative subtype. Patients with triple-negative breast cancer in prognostic stage I and II accounted for $17.7 \%$; the prognostic stage III and IV was $82.7 \%$. The prognostic stage I and II proportion accounted for $52 \%$, and prognostic stage III

Table 3. Change of TNM Stage and Prognostic Stage

\begin{tabular}{|c|c|c|c|}
\hline \multicolumn{2}{|c|}{ TNM stage } & \multicolumn{2}{|c|}{ Prognostic stage } \\
\hline Stage & $\mathrm{N}$ & Stage & N (\%) \\
\hline \multirow[t]{3}{*}{ IA } & 188 & IA & $12(66.5 \%)$ \\
\hline & & IB & $42(22.3 \%)$ \\
\hline & & IIA & $21(11.2 \%)$ \\
\hline \multirow[t]{4}{*}{ IIA } & 243 & IB & $13(57.2 \%)$ \\
\hline & & IIA & $28(11.5 \%)$ \\
\hline & & IIB & $32(13.2 \%)$ \\
\hline & & IIIA & $44(18.1 \%)$ \\
\hline \multirow[t]{6}{*}{ IIB } & 157 & IB & $17(10.8 \%)$ \\
\hline & & IIA & $1(0.6 \%)$ \\
\hline & & IIIA & $25(15.9 \%)$ \\
\hline & & IIIB & $23(14.6 \%)$ \\
\hline & & IIIC & $14(8.9 \%)$ \\
\hline & & Unknown & $77(49.0 \%)$ \\
\hline \multirow[t]{6}{*}{ IIIA } & 102 & IB & $14(13.7 \%)$ \\
\hline & & IIA & $4(3.9 \%)$ \\
\hline & & IIB & $59(57.8 \%)$ \\
\hline & & IIIA & $11(10.7 \%)$ \\
\hline & & IIIB & $10(9.8 \%)$ \\
\hline & & IIIC & $4(3.9 \%)$ \\
\hline \multirow[t]{2}{*}{ IIIB } & 7 & IIIB & $3(42.9 \%)$ \\
\hline & & IIIC & $4(57.1 \%)$ \\
\hline \multirow[t]{3}{*}{ IIIC } & 67 & IIIA & $2(3.0 \%)$ \\
\hline & & IIIB & $31(46.3 \%)$ \\
\hline & & IIIC & $34(50.7 \%)$ \\
\hline IV & 20 & IV & $20(100 \%)$ \\
\hline
\end{tabular}


Table 4. Distribution of Prognostic Stage and Subtype

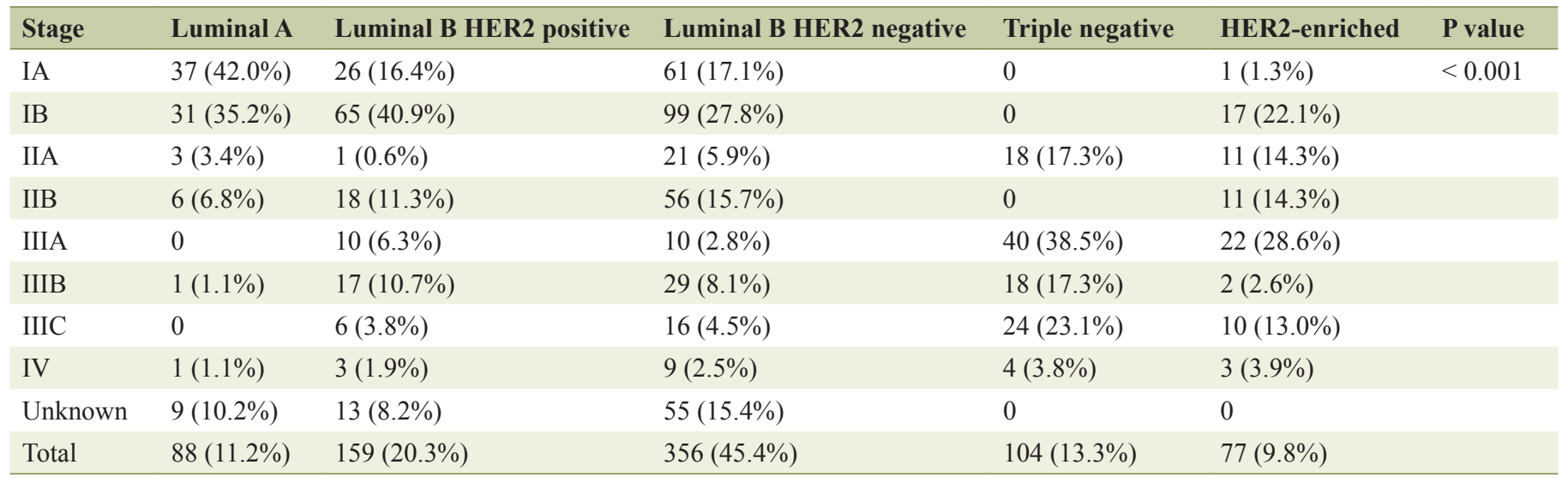

and IV proportion accounted for $48 \%$ in HER 2 positive subtype (Table 4).

Base on the anatomic stage, the prognostic stage system of the breast cancer is more useful and more rigorous for clinicians to understand the biological characteristics of breast cancer. Besides of intrinsic subtypes and anatomic stage, the histological grade $(\mathrm{G})$ was the only factor in the evaluation of prognosis. Therefore, we should pay more attention to accurate histological grading evaluation. AJCC 8th edition also recommended histological grading process as the prior category. In clinical course, histological grade III is rare in patients with luminal A breast cancer who had definitely better outcomes compared to other intrinsic subtypes. Most of the prognostic stage of luminal A breast cancer downstaged [12]. However, most of the triple negative and HER2 positive subtype upstaged in prognostic stage due to poor differentiation and high risk of recurrence and metastasis [13]. The prognostic factors were too complicated to evaluate the prognostic stage of luminal B, and more researches are needed to understand how to accurately predict the outcome of luminal B breast cancer [14]. Thus, the treatment of different subtypes could be adjusted by prognostic stage system based on anatomic stage.

\section{Acknowledgments}

We wish to thank our colleagues at the Department of Pathology for their technical support.

\section{Conflicts of Interest}

The authors declare that they have no conflicts of interest.

\section{References}

1. Amin MB, Edge S, Greene FL. AJCC Cancer Staging Manual 8th ed. New York. Springer, 2016.

2. Goldhirsch A, Wood WC, Coates AS, Gelber RD, Thurlimann B, Senn HJ. Strategies for subtypes - dealing with the diversity of breast cancer: highlights of the St. Gallen International Expert Consensus on the Primary Therapy of Early Breast Cancer 2011. Ann Oncol. 2011;22(8):17361747.

3. Hammond ME, Hayes DF, Wolff AC, Mangu PB, Temin $\mathrm{S}$. American society of clinical oncology/college of american pathologists guideline recommendations for immunohistochemical testing of estrogen and progesterone receptors in breast cancer. J Oncol Pract. 2010;6(4):195197.

4. Wolff AC, Hammond ME, Hicks DG, Dowsett M, McShane LM, Allison KH, Allred DC, et al. Recommendations for human epidermal growth factor receptor 2 testing in breast cancer: American Society of Clinical Oncology/ College of American Pathologists clinical practice guideline update. Arch Pathol Lab Med. 2014;138(2):241-256.

5. Elston CW, Ellis IO. Pathological prognostic factors in breast cancer. I. The value of histological grade in breast cancer: experience from a large study with long-term follow-up. Histopathology. 1991;19(5):403-410.

6. Edge SB, Compton CC. The American Joint Committee on Cancer: the 7th edition of the AJCC cancer staging manual and the future of TNM. Ann Surg Oncol. 2010;17(6):1471-1474.

7. NCCN Guidelines from the NCCN home page: https:// www.nccn.org/professionals/physician_gls/pdf/breast. pdf.

8. Van Poznak C, Somerfield MR, Bast RC, Cristofanilli M, Goetz MP, Gonzalez-Angulo AM, Hicks DG, et al. Use of Biomarkers to Guide Decisions on Systemic Therapy for Women With Metastatic Breast Cancer: American Society of Clinical Oncology Clinical Practice Guideline. J Clin Oncol. 2015;33(24):2695-2704.

9. Edge SB, Byrd DR, Compton CC, Fritz AG, Greene FL, Trotti A. AJCC Cancer Staging Manual. 7th ed. New York: Springer. 2010.

10. Puig-Vives M, Sanchez MJ, Sanchez-Cantalejo J, Torrella-Ramos A, Martos C, Ardanaz E, Chirlaque MD, et al. Distribution and prognosis of molecular breast cancer subtypes defined by immunohistochemical biomarkers in a Spanish population-based study. Gynecol Oncol. 
2013;130(3):609-614.

11. Retraction: Luminal B tumors are the most frequent molecular subtype in breast cancer of North African women: an immunohistochemical profile study from Morocco. Diagn Pathol. 2013;8:127.

12. Agarwal G, Nanda G, Lal P, Mishra A, Agarwal A, Agrawal V, Krishnani N. Outcomes of Triple-Negative Breast Cancers (TNBC) Compared with Non-TNBC:
Does the Survival Vary for All Stages? World J Surg. 2016;40(6):1362-1372.

13. Siegel RL, Miller KD, Jemal A. Cancer statistics, 2015. CA Cancer J Clin. 2015;65(1):5-29.

14. Mazouni C, Rimareix F, Mathieu MC, Uzan C, Bourgier $\mathrm{C}$, Andre F, Delaloge S, et al. Outcome in breast molecular subtypes according to nodal status and surgical procedures. Am J Surg. 2013;205(6):662-667. 